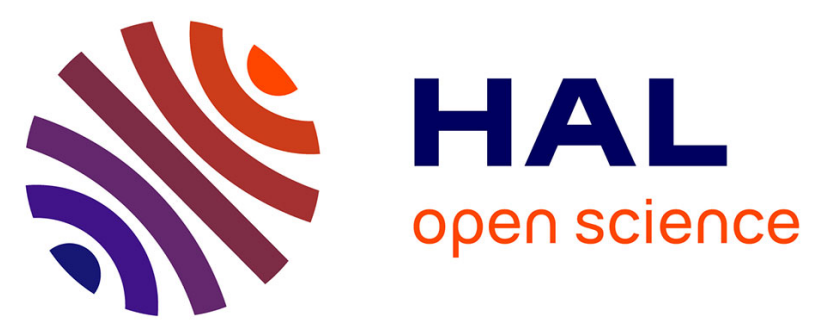

\title{
Evolutions and Improvements in CFOSAT SWIM Products
}

\author{
C. Tourain, Danièle Hauser, Dunya Alraddawi, L. Hermozo, R. Rodriguez \\ Suquet, Patricia Schippers, L. Aouf, A. Dalphinet, Christophe Dufour, J-M. \\ Lachiver, et al.
}

\section{To cite this version:}

C. Tourain, Danièle Hauser, Dunya Alraddawi, L. Hermozo, R. Rodriguez Suquet, et al.. Evolutions and Improvements in CFOSAT SWIM Products. IGARSS 2021 - 2021 IEEE International Geoscience and Remote Sensing Symposium, Jul 2021, Brussels, Belgium. pp.7386-7389, 10.1109/IGARSS47720.2021.9553274 . insu-03379024

\section{HAL Id: insu-03379024 https://hal-insu.archives-ouvertes.fr/insu-03379024}

Submitted on 18 Oct 2021

HAL is a multi-disciplinary open access archive for the deposit and dissemination of scientific research documents, whether they are published or not. The documents may come from teaching and research institutions in France or abroad, or from public or private research centers.
L'archive ouverte pluridisciplinaire HAL, est destinée au dépôt et à la diffusion de documents scientifiques de niveau recherche, publiés ou non, émanant des établissements d'enseignement et de recherche français ou étrangers, des laboratoires publics ou privés. 


\title{
EVOLUTIONS AND IMPROVEMENTS IN CFOSAT SWIM PRODUCTS
}

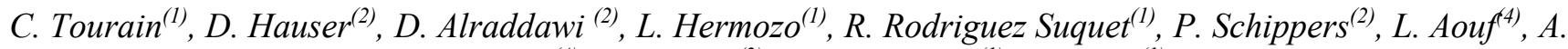

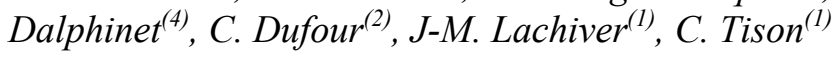

(1) CNES, 18 avenue Edouard Belin, 31400 Toulouse, France

(2) LATMOS, CNRS, UVSQ, Sorbonne Université, 11, boulevard d'Alembert, 78280 Guyancourt, France

(3) ACRI-ST, 260 Route du Pin Montard, 06904 Biot, France

(4) Météo France, 42 Avenue Gaspard Coriolis, 31057 Toulouse, France

\begin{abstract}
The Chinese-French oceanography satellite, CFOSAT, was launched on October 2018. Two Ku-band scatterometers are on-board: SCAT for the wind observation and SWIM for the wave observation. After a first phase mainly dedicated to validation and identification of improvement possibilities, the ground processing and products generated were upgraded. This paper presents the main evolutions implemented and their positive impacts on the SWIM data quality.
\end{abstract}

Index Terms - radar, ocean waves, scatterometer, CFOSAT, SWIM

\section{INTRODUCTION}

The CFOSAT mission, launched on October, $29^{\text {th }} 2018$, is composed of two radar sensors both scanning in azimuth: SCAT, a fan-beam wind scatterometer [1], and SWIM designed for wave measurements [2]. Thanks to a fruitful Chinese-French cooperation, this mission opens a new era in oceanography, allowing the combined measurement and monitoring of wind and waves.

With its collocated measurements of ocean surface wind and waves, CFOSAT provides very useful observations for improving atmospheric and oceanographic models and predictions, and for better understanding processes at the ocean surface and ocean/atmosphere interactions.

This paper focuses on the SWIM measurements. SWIM is an innovative $\mathrm{Ku}$-band real-aperture wave scatterometer, with 6 low-incidence rotating beams [2]. An important task of CALibration and VALidation (CAL/VAL hereafter) on the instrument and products was performed at the beginning of the mission [3]. This work allowed to well characterize after the launch, the performance and product quality of this novel instrument. It showed performances for the nadir beam products at the same level as conventional altimeters [4] [5], in spite of the SWIM instrument lower sampling rate of mean waveforms $(4.5 \mathrm{~Hz}$ vs $20 \mathrm{~Hz})$. Concerning products from offnadir observations, it highlighted promising results and also areas of improvements. Here we synthesize the main evolutions recently applied to the data processing, and the consecutive improvements observed in the product quality.

Evolutions are mainly oriented to improve the wave slope spectra retrieval. Starting with a work on the along track speckle noise to mitigate its impact, then a new Modulation Transfer Function (MTF) has been chosen to reduces some biases observed.

\section{ALONG TRACK SPECKLE NOISE MITIGATION}

It was shown in [3] that within an angular sector of about $\pm 15^{\circ}$ on each side of the along track direction, there is a strong increase in the energy level, not related to the surface ocean waves. This is due to the decrease of Doppler bandwidth for measurement performed in this sector: the number of uncorrelated echoes reduce drastically, so the integration of received signals is less efficient to reduce the speckle noise. This phenomenon was expected, but not taken into account in the initial processing versions. This phenomenon affects the wave slope spectra inverted from the signal modulation spectra, and consequently induces errors on the wave parameters.

For directions far from along track, observations are compliant with theory: speckle noise has a shape and level constant with azimuth, decreasing almost linearly with the wave number, and shows no sea state dependency. Its level is directly related to the number of samples used in the backscattered waveform average. To take into account the real impact of the speckle noise within the $\pm 15^{\circ}$ sector and also to apply better corrections of the speckle noise background level outside this sector, an empirical speckle model was established by analyzing the SWIM signal spectra in conditions of minimum impact of ocean waves [3]. One specificity of this model is that in the sector close to the along-track direction $\left( \pm 15^{\circ}\right)$, the density spectrum of speckle noise varies with azimuth direction, latitude and sea-state conditions. This model is implemented in the processing chain since 2020, June the $24^{\text {th }}$. The strong energy in the 0 $15^{\circ}$ area, due to speckle noise, is largely reduced, this allows to provide the whole wave slope spectra in the product. We also observe a positive impact on wave parameters. Figure 1 shows the wave parameters from SWIM products compared 
to parameters from MFWAM model, with the previous and the new speckle correction. We observe a better consistency, with a reduction of the bias in significant wave height and a smaller scatter.
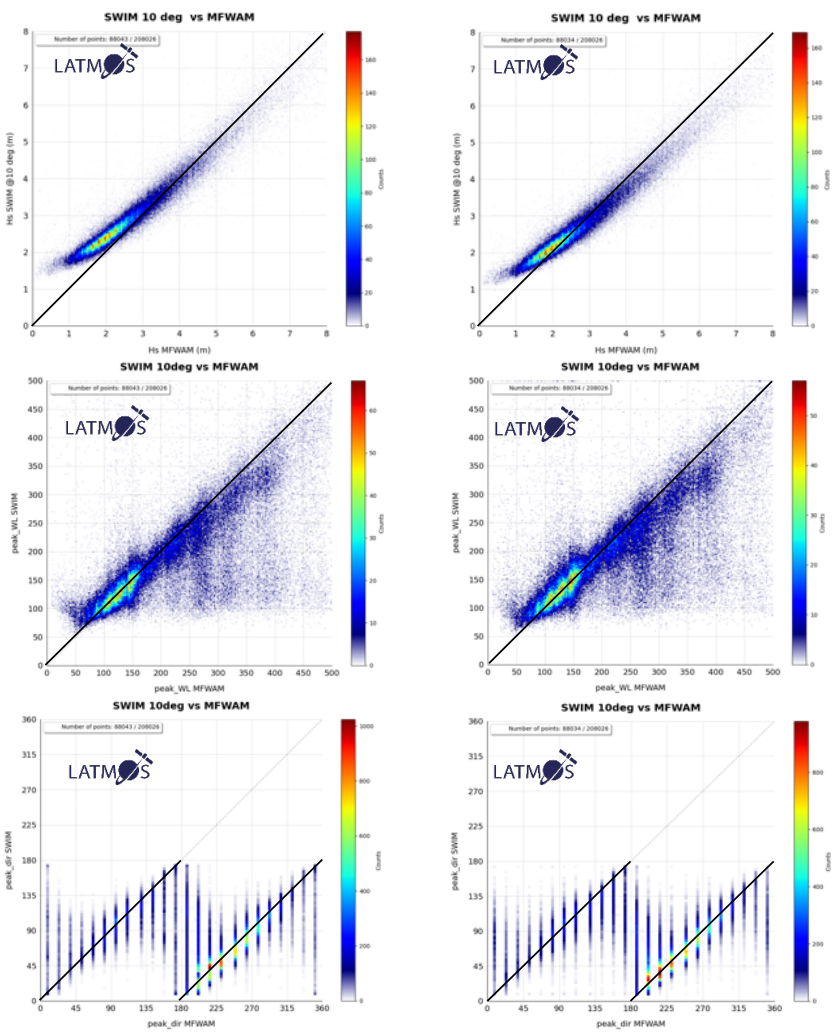

Figure 1 : histogram of wave parameters from CFOSATSWIM (beam $10 \mathrm{deg}$ ) vs MFWAM wave parameters ( 2019 April $26^{\text {th }}-08^{\text {th }}$ May), with the previous speckle noise correction (left), and the new speckle noise correction

(right). Top: significant wave height; middle: peak wavelength; bottom: dominant direction.

\section{MODULATION TRANSFER FUNCTION MODIFICATION}

The wave slope spectrum is obtained via the transformation of the directional modulation spectra thanks to a so called modulation Transfer Function (MTF). This MTF is a proportionality coefficient between the signal modulation spectrum and the wave slop spectrum. Several $\mathrm{MTF}_{\mathrm{s}}$ are implemented in the ground segment and any of them can be activated for the operational processing.

The initial MTF applied in operational product generation (until 2020 October $12^{\text {th }}$ ) was the so called MTF1, expressed by (1)

$$
\operatorname{MTF}(\theta, \phi)=\frac{\sqrt{2 \pi}}{L_{y}} \alpha^{2}(\theta)
$$

Where $\boldsymbol{L}_{\boldsymbol{y}}$ is the azimuth length of the footprint, $\boldsymbol{\alpha}$ a sensitivity factor $=\operatorname{cotan}(\theta)-\frac{1}{\sigma_{0}} \frac{\partial \sigma_{0}}{\partial \theta}, \boldsymbol{\theta}$ the incidence angle, and $\sigma_{0}$ the normalized radar cross-section.

In this case, the sensitivity factor $\alpha$ was estimated from the $\sigma_{0}$ measurements thanks to a fit on the mean profile of $\sigma_{0}(\theta)$ constructed over all the SWIM incidences $\left(0-10^{\circ}\right)$. The MTF1 is given for each azimuth bin (every $15^{\circ}$ ) and each spectrum beam $\left(6^{\circ}, 8^{\circ}\right.$ and $\left.10^{\circ}\right)$ for each box (right and left side of the nadir track). During the CAL/VAL phase, it was shown that the significant wave height $(\mathrm{SWH})$ resulting from this normalization method was biased compared to the nadir significant wave height, and to buoy measurements and model outputs (see also top right panel of Fig.1). This bias was maximum and positive at small significant waves height (below 2m) and was negative at high significant wave heights. This was attributed to the uncertainty remaining in the shape of the mean profiles of $\sigma_{0}(\theta)$ which combine the information from the 6 independent beams of SWIM.

In order to avoid these biases in significant wave heights, it was decided to switch the operational processing to the so called MTF3 expressed by (2), on 2020 October the $12^{\text {th }}$. In this case, the MTF is determined for each spectrum beam $\left(6^{\circ}\right.$, $8^{\circ}$ and $10^{\circ}$ ) and for each box (no azimuth dependency). It uses the SWH obtained from the nadir beam to normalize the energy of the wave height spectrum.

$$
M T F=\left(\frac{4}{S W H}\right)^{2} \iint P_{m}(k, \phi) \frac{1}{k} d k d \phi
$$

With $\boldsymbol{P}_{\boldsymbol{m}}$ the signal modulation spectrum corrected from speckle noise effects, $\boldsymbol{k}$ the wavenumber and $\boldsymbol{\phi}$ the azimut angle. This version of MTF (called MTF3) constrains the wave height spectrum to provide a SWH consistent with the nadir measurements. The SWH from CFOSAT/SWIM nadir beam shows the same level of performance as altimetry missions [5]. Thanks to the normalization of the energy spectrum with this nadir SWH, the wave parameter SWH form spectrum beams processed with MTF3 is more consistent with models (ECMWF, MFWAM). The overestimation at low wave heights almost disappears, the underestimation at high wave heights is also largely limited, while preserving the azimuth and wavelength directions. Figure 2 shows the better consistency between CFOSAT/SWIM wave parameter SWH and MFWAM SWH. Figure 3 shows that the same improvement is obtained in the comparison of CFOSAT/SWIM wave parameter SWH with in situ data given by NDBC buoys. Other wave parameters wavelength and direction are almost unchanged by application of MTF3, they keep the level of performance shown in Figure 1. Beside these improvements, it has to be noted that it presents a limitation. The wave slope spectrum is constructed over a cell (so called box) of $70 \mathrm{~km}$ by $90 \mathrm{~km}$ on each side of the sub satellite track; with MTF3, wave slope spectrum energy is normalized with a SWH value from the sub satellite track, which can be in some cases not fully representative for the whole cell. 

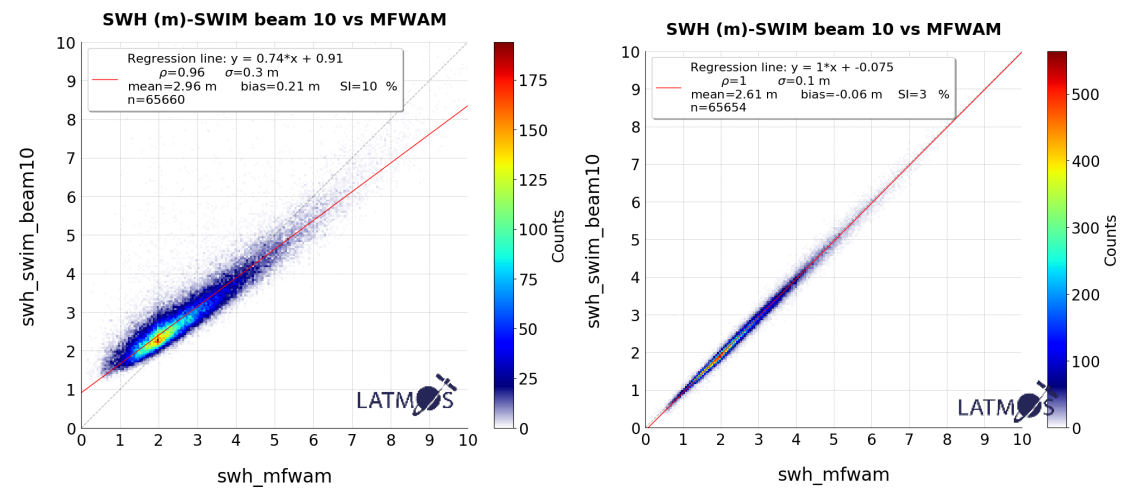

Figure 2 SWIM wave parameter SWH from $10^{\circ}$ beam data compared to MFWAM SWH, for MTF1 (left and MTF3 (right). The period of observations is 26 January 2020 to 6 February 2020
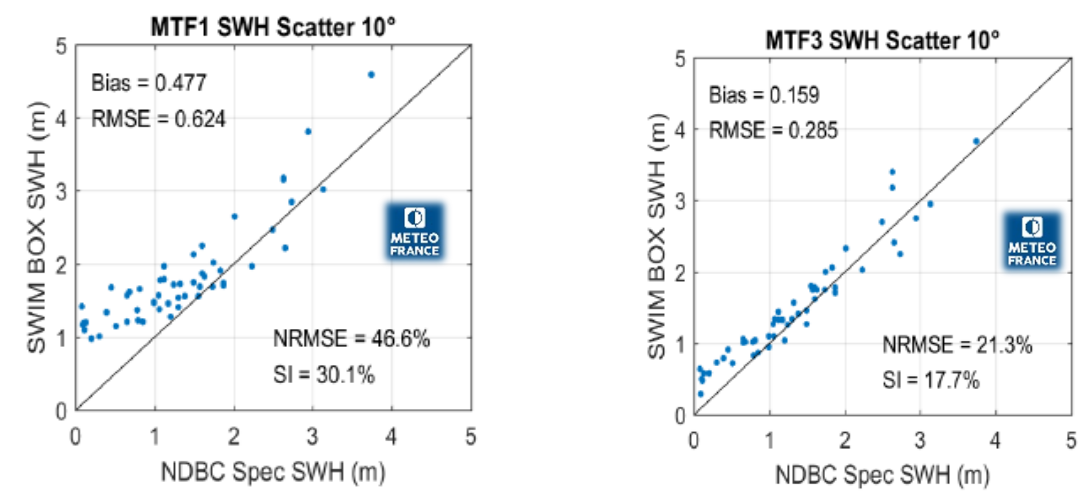

Figure 3 SWIM wave parameter SWH from $10^{\circ}$ beam data compared to NDBC buoys SWH, for MTF1 (left and MTF3 (right) for period from 2020, January the 25th to February the 7th.

\section{PLANNED EVOLUTION AND PERSPECTIVES}

In complement to evolutions implemented and presented in this paper, several areas of improvements are continuously investigated. One is on the mitigation of spurious peaks observed in 1D wave height spectra in particular for low seastate conditions. These spurious peaks are due the amplification of the noise floor at small wave numbers when converting SWIM wave slope spectra to wave height spectra. Even if the speckle correction is appropriate in the mean, some variability of speckle noise induces non-zero values of the noise floor [4]. When long waves are present, this noise contribution is negligible compared to the wave energy, but otherwise (and mainly for low sea-state conditions), after conversion in wave height spectra, this problem generates spurious peaks in the height spectra, which hampers to use these height spectra to characterize the peak wavelength (or peak frequency). A filtering method of these peaks is currently under tuning. The challenge is to filter the energy of the noise at these small wavenumbers (long wavelengths) while keeping the energy when real long waves (swell) are present. The method under tuning consist in i) choosing a wavenumber limit (called $k_{\text {filt }}$ currently chosen as 0.025 $\mathrm{rad} / \mathrm{m}$ ), ii) comparing the peak wavenumber $\mathrm{k}_{\text {peak2 } 2 \mathrm{Ds}}$ from the 2D slope spectra (not affected by these parasitic peaks) to $\mathrm{k}_{\text {filt, }}$ iii) only when $\mathrm{k}_{\text {peak2Ds }}$ is larger than $\mathrm{k}_{\text {filt }}$ (wavelengths of waves smaller than $2 \pi / \mathrm{k}_{\text {filt }}$, impose a zero value to the $2 \mathrm{D}$ wave slope spectrum energy for all $\mathrm{k}$ less than $\mathrm{kffilt}_{\text {, }}$ iv) calculating the omni-directional wave height spectrum form this modified 2D slope spectrum.

Figure 4a-b show histograms of the differences of peak wavelengths between SWIM and MFWAM as a function of the significant wave height. In Fig. (a) the peak wavelength of SWIM is calculated from the omni-directional wave spectrum without any correction, whereas in (b) it is estimated with our filtering method. The figures show that the important positive biases with respect to the MFWAM values observed mainly at wave heights smaller than $2.5 \mathrm{~m}$ are significantly reduced by using the filtering method.

As already mentioned in [4], another improvement shall result from a better account of non-homogenous scenes. A method to reject the signal from those scenes has been considered, based on a maximum value of $\sigma_{0}$ variances estimated over each footprint after filtering the fluctuation due to waves (scales smaller than $500 \mathrm{~m}$ ). A complementary work was performed to refine the filtered $\sigma_{0}$ variance parameter, an analysis of all products will be performed after the reprocessing campaign to determine the pertinent threshold for rejection. 
(a)

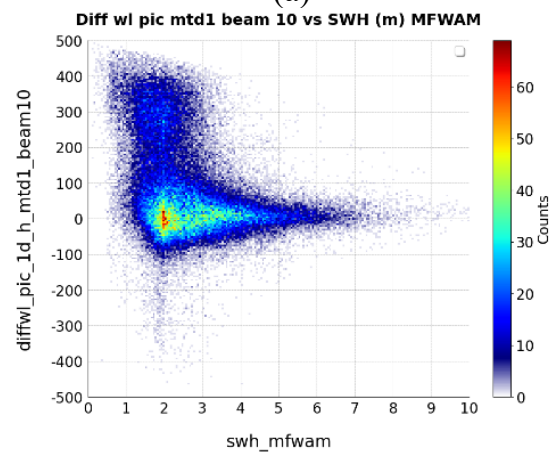

(b)

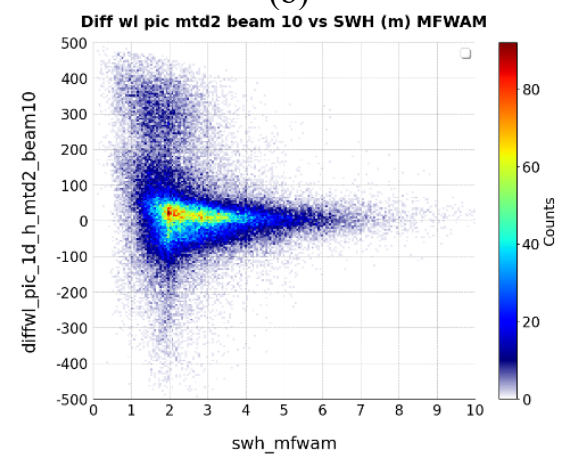

Figure 4 Histogram of the differences between SWIM and MFWAM peak wavelengths as a function of significant wave height. (a) when the SWIM peak wavelengths is estimated on the omni-directional wave height spectrum without any prior filtering (b) same but with filtering on the 2D wave slope spectra. The data set is from 2020-01-26 to 2020-02-06, processed with the version V5.0.

Further analyses are also conducted on modulation transfer function. As described in section 2, the MTF3, while showing a better consistency with model and buoy data for SWH, presents some limitations, mainly the normalization of wave slope spectrum energy with a non co-localized parameter, i.e. the SWH from the nadir beam. The MTF1 is further studied to make its derived SWH consistent with model and buoys data, and use this co localized MTF. The analysis and the fit the mean profile of $\sigma_{0}(\theta)$ will be reviewed to better estimate the sensitivity factor $\alpha$.

An evolution is also considered thanks to observations of data acquired for two years. In the current CFOSAT-SWIM product, the direction wave parameter is given with a $180^{\circ}$ ambiguity. Based on up to down wave asymmetry observed in the SWIM fluctuation spectrum a potential removal of this ambiguity method is proposed [5], this method will be analyzed in terms of performance and applied if conclusive.

\section{CONCLUSIONS}

After two years in flight, the interest of SWIM products has been shown. Evolution of the data processing described here has enhanced performances of the disseminated product. As explained, the new speckle noise correction allows to provide the whole spectrum spectra. Combined with the application of the new modulation transfer function MTF3, wave parameters estimated from the wave spectra now show an improved agreement with model and buoy data, mainly for the SWH. With the level of quality raised now, CFOSAT-SWIM products are reliable and their assimilation shows potential improvement in many applications, such as wave forecasting, at global level [7] and for specific areas such as Southern Ocean [8]. As SWIM is a very innovative instrument, there are still areas of improvement and perspectives for new applications. Work keeps going on identified subjects, and analyses are continuously performed to exploit all the potential of SWIM data.

\section{REFERENCES}

[1] Liu Jianqiang, Wenming Lin, Xiaolong Dong, et al, «First Results From the Rotating Fan Beam Scatterometer Onboard CFOSAT », 10.1109/TGRS.2020.2990708, 2020

[2] Hauser D., C. Tison, T. Amiot, L. Delaye, N. Corcoral et al, SWIM: the first spaceborne wave scatterometer, 10.1109/TGRS.2017.2658672, 2017

[3] Hauser D., C. Tourain, L. Hermozo et al, "New observations from The SWIM radar on board CFOSAT; instrument validation and ocean wave measurement assessment", doi 10.1109/TGRS.2020.2994372, 2020

[4] Tourain C., Hauser D., Hermozo L., Rodriguez-Suquet R., Schippers P., et al., CAL/VAL PHASE FOR THE SWIM INSTRUMENT ONBOARD CFOSAT. IEEE International Geoscience and Remote Sensing Symposium (IGARSS), Sep 2020, Virtual, United States.

[5] Tourain C., F. Piras, A. Ollivier et al, "Benefits of the Adaptive algorithm for retracking altimeter nadir echoes: results from simulations and CFOSAT/SWIM observations", IEEE/TGRS, under review, 2020

[6] Huimin Li, D. Hauser, B. Chapron, F.Nouguier, P. Schippers, Biao Zhang, Jinsong Yang and Yijun He, «Up-to-downwave asymmetry of the CFOSAT SWIM fluctuation spectrum for wave direction ambiguity removal, submitted to IEEE TGRS, 2020

[7] Aouf L., Hauser D., Chapron D., Toffoli A., Tourain C., Peureux C., "New directional wave satellite observations: Towards improved wave forecasting and climate description in Southern Ocean', Geophysical Research Letters, under review, 2020

[8] Wang J. K., Aouf L., Dalphinet A., Xu Y., J. Q. Liu J. Q., "Acquisition of Significant Wave Height from the CFOSAT SWIM Spectra through Deep Neural Network: Impact on Assimilation in Wave Model',, Journal of Geophysical Research Oceans, under review, 2020 